\title{
La cognición en la web a través de un chatbot como elemento disruptivo
}

\section{The cognition on the web through a chatbot as a disruptive element}

\author{
PÉREZ-CALDERÓN, Ricardo†* \& SUAREZ-JACOBO, Martin \\ Universidad Politécnica del Valle de México \\ ID $1^{\text {er }}$ Autor: Ricardo, Pérez-Calderón / ORC ID: 0000-0002-8320-1769, CVU CONCYT ID: 3530003 \\ ID $1^{\text {er }}$ Coautor: Martin, Suarez-Jacobo / ORC ID: 0000-0002-3903-9469, CVU CONACYT ID: 68728
}

DOI: $10.35429 / J C T .2019 .11 .3 .7 .17$

Recibido 19 de Octubre 2019, Aceptado, 05 de Diciembre, 2019

\begin{abstract}
Resumen
Hoy en día las empresas buscan satisfacer la mayoría de las necesidades de sus clientes y el uso de la tecnología ayudaría a resolverlas y a tener una comunicación eficiente en tiempo real con ellos. Por lo tanto, el objetivo de este proyecto, es realizar una herramienta tecnológica disruptiva, un chatbot y hacer un análisis de su empleo, y que a través del uso de una red social (Facebook) puede romper paradigmas de comportamiento en una cantidad masiva de usuarios y con ello maximizar el impacto social que tiene toda actividad dentro de Internet. Lo importante es que la imagen comercial que pueda dejar a los usuarios una herramienta tecnológica como ésta (chatbot) sea de completa credibilidad y satisfacción a los clientes de las empresas que aplican esta tecnología. Adicionalmente, es bien conocido que las generaciones de hoy en día no viven sin las redes sociales y a ellos les es mucho más práctico adquirir productos a través del Internet. Por último, ya hay empresa que hacen uso de esta tecnología, un chatbot: Aeroméxico, National Geographic, Starbucks, Next_u, entre otras.
\end{abstract}

Cognitivo, Chatbot, Disruptiva

\begin{abstract}
Today, the companies try to basic needs of their clients, the use of technology would help solve and have efficient communication in real time with them. Therefore, the objective of this project is to perform a disruptive technological tool, a chatbot, and make an analysis of your employment in uses a social network (Facebook), to break paradigms of behavior in a massive number of users and that maximizes the social The main subject is the commercial impact, complete credibility and satisfaction this tool in the user (around the company that apply chatbot). Nowadays, companies seek to satisfy most of their client's needs; that tool assistance to reduce the troubles between them and the company. Moreover it is well known that today's generations do not live without social networks interactions and even more so that they are buying products through the internet. Finally, there are companies that make use of this technology: Aero México, National Geographic, Starbucks, Next_u, etc.
\end{abstract}

Cognitive, Chatbot, Disruptive

Citación: PÉREZ-CALDERÓN, Ricardo \& SUAREZ-JACOBO, Martin. La cognición en la web a través de un chatbot como elemento disruptivo. Revista de Tecnología Informática. 2019 3-11: 7-17 


\section{Introducción}

Los procesos cognitivos que los seres humanos experimentan, son la esencia de su adaptación a cada uno de sus roles en donde se desenvuelven. Gómez Zaira (2015), da una definición de cognición "son procesos internos como el aprendizaje, el razonamiento, la atención, la memoria, la resolución de problemas, la toma de decisiones y el procesamiento del lenguaje”.

Así mismo, indica que en la cognición existe una confirmación de un conjunto de señales que envían información a los procesos. Por otro lado, Ayara, (2018) comenta que "el proceso cognitivo ayudará al individuo a responder a los estímulos del entorno.

La interacción entre el sujeto y el entorno hace posible el desarrollo de las operaciones mentales que conduzcan a la elaboración de respuestas para transformar la realidad".

En términos más claros, la cognición es el desarrollo de habilidades del pensamiento que el ser humano trae desde su nacimiento y que en su mayoría se aplican a circunstancias propias de la vida.

Es por ello, que si se aplican a lo largo de su actuar serán provechosas para él, además de que si esa cognición la llevamos a otro ámbito de uso dentro de Internet, entonces se puede identificar y cambiar las formas tradicionales de comportamiento dentro de ella, incluyendo la forma en que se le presenta la información y por ello cada usuario deberá ser un ente disruptivo que rompa con paradigmas tradicionales de comportamiento en lo tangible y en lo intangible.

\section{La cognición}

Según lo que indica Gómez, (2015), existen 11 niveles de complejidad en las operaciones cognitivas de un usuario cuando él interactúa con algo intangible, que van desde el procesamiento de la información hasta el generarse juicios de valor en el espacio socio cognitivo, en ese sentido Shedron, (1994), quien fue citado por la misma autora, indica que el "diseño de interacción de la información es la confluencia de pautas de varios diseños, como el de la información, el de interacción y el sensorial".
La figura 1, esquematiza la interacción de estos tres tipos de diseños y la intersección de ellos. Shedron, (2014), los describe de manera puntual.

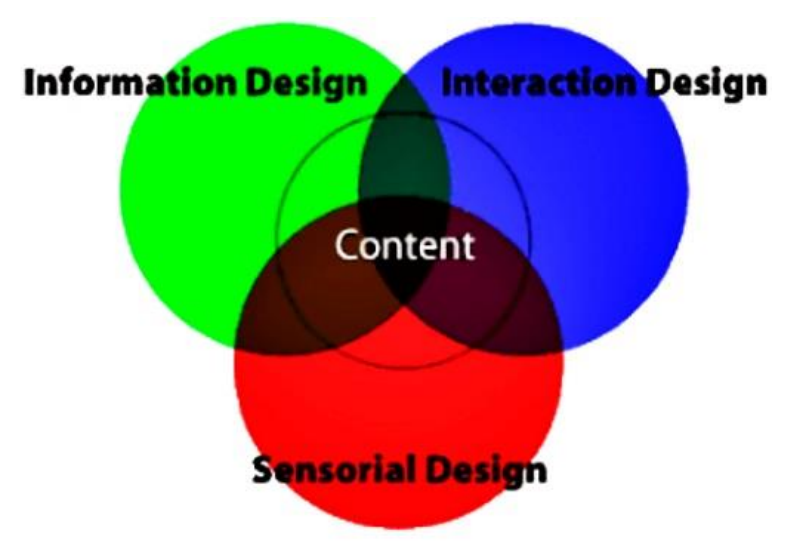

Figura 1 Information Interaction Design: A Unified Field Theory of Design, (Shedron, 2014)

1. Information Design. El Diseño de Información, se centra principalmente en la representación de datos y su presentación.

2. Interaction Design. El Diseño de Interacción, es la creación de experiencias atractivas, es esencialmente la creación de historias y narración, es a la vez arte antiguo y nueva tecnología. Es un componente muy crítico para los productos interactivos.

3. Sensorial Design. El diseño Sensorial, simplemente es el empleo de todas las técnicas con las que nos comunicamos con otros a través de nuestros sentidos (sentir, oír y oler), son muy válidos y enriquecedores.

Adicionalmente, también Shedron indica lo siguiente, "Estos niveles de comprensión son importantes porque definen los límites con los que podemos crear y comunicar".

Cada uno de estos diseños generan un proceso cognitivo en los usuarios al momento de interactuar con cualquier plataforma $o$ aplicación.

Por otro lado, Campos, (2004), indica que a pesar de los avances en la investigación cognoscitiva es todavía muy generalizado el conocimiento en los procesos sociales y a su vez indica que el modelo constructivista, ha permeado la investigación en esta área de especialización de lo cognoscitivo y que no se ha alcanzado a la práctica educativa concreta. 
En términos precisos, este autor describe que son formas racionales con los que nos relacionamos y que se desprenden varios conceptos de la lógica del conocimiento formal, como los siguientes: clasificar, analizar, relacionar, inferir e interpretar y que la base de la lógica informal es un sincretismo ya que se basa en reglas formales y concretas, incidiendo en el resultado esperado. Por lo tanto, la lógica formal puede ser altamente creativa en situaciones nuevas y la lógica informal depende de la experiencia y de las reglas de producción individuales.

Aunado a lo anterior, la actividad de la memoria es un proceso altamente ligado a lo cognitivo del ser humano, y que sin embargo, si las premisas de su funcionamiento son manipuladas entonces los resultados de ese raciocinio son muy variados.

Por ejemplo en la Revista Digital Infocop de España en el 2011, se escribió un artículo del efecto de Google en la memoria con resultados muy variados, los siguientes investigadores Betsy Sparrow de la Universidad de Columbia, Jenny Liu de la Universidad de Wisconsis-Madison y Daniel M. Wegner de la Universidad de Harvard, ellos llevaron a cabo un experimento y lo explican de la siguiente manera, "consistía en un estudio experimental en el que se solicitaba a los participantes que prestaran atención a diferentes informaciones de contenido curioso y novedoso.

Tras la presentación de los estímulos informativos, se les ofrecía la posibilidad de escribir dicha información en el ordenador y almacenarla. A partir de aquí, a un grupo de participantes se les indicó que la información registrada iba a borrarse, a otro que iba a guardarse en un archivo de fácil acceso y a un tercer grupo que se quedaría en archivo de difícil acceso.

Al solicitarles que recordaran la información presentada, los resultados fueron de acuerdo a las premisas (borrarse, guardarse y difícil acceso) que se les indicaron al inicio del experimento". Por lo tanto, cuando al ser humano se le predispone, su juicio cognitivo también se verá afectado.
Por último, Vergara, (2008) en su curso de psicología social indica que "el mundo de la vida cotidiana es aquel que se da por establecido como realidad, el sentido común que lo constituye se presenta como la realidad por excelencia", adicionalmente mencionan que, "la realidad de la vida cotidiana es una construcción intersubjetiva, un mundo compartido, lo que presupone procesos de interacción y comunicación mediante los cuales comparte con los otros y experimenta a los otros. Es una realidad que se expresa como mundo dado, naturalizado, por referirse a un mundo que es común a muchos hombres.

Se aprende del otro, a los sucesos, acontecimientos y objetos por medio de esquemas tipificadores, que se vuelven progresivamente anónimos en la medida que se alejan del aquí y del ahora, de la situación cara a cara; es decir, que el conocimiento se construye y reconstruye no sólo con y mediante los semejantes, con quienes se establece interacción directa, sino con los contemporáneos y no sólo con ellos sino con los antecesores y sucesores. Los que me han precedido y me sucederán en la historia total de la sociedad". Por lo tanto, la adaptación de los seres humanos a sus roles en donde se desenvuelven, dependen de todo lo que les acontece.

Para concluir, los procesos cognitivos son los que dan la capacidad de dar atención individual o colectiva, entonces, como podrían empotrarse a través de la tecnología, es decir, el uso de Internet y la implementación de Inteligencia Artificial (dispositivos que intentan pensar como un ser humano) para dar soluciones de esa atención en tiempo real a peticiones de los clientes en las organizaciones. Lo que se busca, como comenta Aliaga, (2019) es que, "los comercios online traten de ser cada vez más personales y cercanos a los clientes." eso es el planteamiento del proyecto, implementar una herramienta On line en las redes sociales.

Según el diario el economista de España, en el año 2016, dijo lo siguiente en relación a los chatbots, "Hoy en día las apps de mensajería están convirtiéndose en uno de los servicios más utilizados por los usuarios de dispositivos móviles. Por esta razón, varios sectores están incorporando estos canales a sus modelos de negocio, un servicio que no estará controlado por personas, sino por robots". 
Ejemplos de empresas de gran renombre que ya usan esta tecnología son: el Washington Post, la empresa Booking.com, Expedia.com, Cortana de Microsoft, KLM una empresa holandesa, y la plataforma Outbrain, el chatbot según el diario el economista de España debe servir como aquel elemento para que "no dañe la credibilidad" y lo que a futuro se pretende con un chatbot es que funcione como si fuera un reportero

Charlan, J, (2018), publicó en la página web Rethink de ESIC, esta plataforma es un proyecto muy ambicioso sobre marketing, management, economía digital, comercial y ventas, escrito por reconocidos expertos; dice que los chatbots "son bots especializados y creados para mantener conversaciones y ofrecer respuestas preconcebidas.

Por lo tanto, un chatbot es un software que utiliza mensajes estructurados para emitir respuestas desde una máquina hacia un interlocutor humano". Adicionalmente indican que lo que buscan las empresas con el uso de un chatbot es un excelente nivel de servicio y calidad.

En la página IAdvise en el 2017, se menciona cómo los chatbot pueden transformar el customer experience online, "Actualmente, la tasa de conversión de las tiendas tradicionales se sitúa alrededor del $20 \%$ al $30 \%$, mientras que el eCommerce, oscila sólo en el $2 \%$. El customer experience rico, visual y palpable es lo que marca la diferencia entre las tiendas tradicionales y las tiendas online: los clientes pueden conversar con los vendedores y con otros clientes. Para garantizar el éxito del eCommerce y rivalizar con los resultados de las tiendas tradicionales, las marcas deben darle un toque humano al customer experience online". Por lo tanto, los chatbots serían el toque humano y una eficiente solución.

Hervouet Julien, (2019), quien es CEO de IAdvizor, publicó en su artículo "The value of online conversations - estimated to generate $€ 7.2$ million benefits for a fast growing online retailer over three years", que el impacto económico de las conversaciones en línea es considerable y que el retorno de inversión para tres años es del 64\%, este estudio se realizó a un minorista europeo. Se contemplaron diversos indicadores y la manera de convertirlos y calcularlos en dinero.
De acuerdo a la inquietud de un gran número de empresas lideres en tecnología, la invovacion y las tendencias del mercado, los chatbots serán de gran auge en futuros años. Por lo tanto, el objetivo de este proyecto es: "Desarrollar e implementar una herramienta tecnológica disruptiva (chatbot) para interactuar con usuarios y con ello romper paradigmas de comportamiento en las redes sociales".

\section{Millenials}

Son la generación conocida como la del Milenio por el año en que nacieron, las fechas de inicio y termino esta generación son variadas pero similares dependiendo de cada autor. Sin embargo la organización. Pew Research Center (2010), indica que ellos, son personas nacidas después de 1980 y que en la actualidad han alcanzado la mayoría de edad. En la figura 2, se ilustran las generaciones de esta organización.

\section{Millennials}

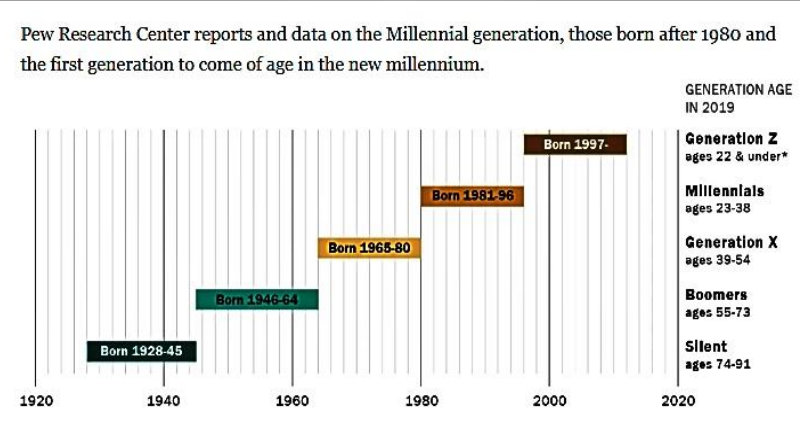

Figura 2 No se ha establecido un punto final cronológico para este grupo. Los rangos de edad de la generación $\mathrm{Z}$ varían según el análisis Pew Research Center

Por lo que indica Gutiérrez, (2014), comenta que los millenials es, "una futura generación de consumidores con nuevas características, necesidades y demandas que conviene conocer por las repercusiones y transformaciones que exigirá a las empresas.”, también indica que son jóvenes entre 20 y 35 años y en su artículo cita a la empresa consultora Delloite quien indica que en el año del 2025 serán el $75 \%$ de la fuerza laboral. Este autor describe de manera precisa seis de las características de ellos:

1. Son Nativos Digitales, se caracterizan por dominar la tecnología como una prolongación de su propio cuerpo. 
2. Multipantalla y multidispositivo. Utilizan múltiples canales y dispositivos digitales para sus actividades e indica que utilizan en promedio 7 horas al día

3. Nomófobos y appdictos. Su vida es móvil y su pantalla principal de entrada a la Red es ya una pantalla móvil. Un $78 \%$ de los Millennials en Latinoamérica posee un móvil (un $10 \%$ más que el año anterior), un $37 \%$ tablet, un $70 \%$ laptop y un $57 \%$ desktop, sociales, sin duda una de las características que mayormente sobresale, ya que al estar en diversas redes sociales es su medio de comunicación.

4. Sociales, son extremadamente sociales. Un $88 \%$ de los millennials latinoamericanos tiene perfiles en redes sociales. No son sólo un medio de comunicación para ellos sino una parte íntegra de su vida social.

5. Críticos y Exigentes. Son mucho más críticos, exigentes y volátiles. De hecho, un $86 \%$ de los consumidores actuales declara que dejaría de hacer negocios con una empresa debido a una mala experiencia de cliente, frente al $59 \%$ de hace 4 años. Y, para los Millennials, las experiencias digitales negativas en línea y móvil tienen un impacto negativo mucho mayor que sobre otros grupos de edad.

6. Exigen personalización y nuevos valores, Los Millennials son clientes que no buscan exclusivamente una buena atención, sino que exigen personalización y esperan que la empresa se adecúe a sus preferencias. Para atender sus demandas, las compañías deben ampliar el conocimiento sobre sus clientes incorporando información social sobre ellos con la idea de aportarles más valor

Por lo anterior, los millenials consideran que sin Internet no podrían subsistir, ellos son el personal que va a dominar la parte laboral en futuros años y que con Internet siempre satisfacen sus necesidades, ellos son los usuarios más pertinentes y con un nivel mayor especialización para poder usar las herramientas automatizadas en línea.
Por lo tanto, este proyecto (Chatbot) será destinado en un alto porcentaje para ellos.

Por último, para Ventura, (2018), indica que un chatbot "es un programa de inteligencia artificial que es capaz de mantener una conversación con un ser humano, dando respuesta a sus preguntas y haciéndoles sugerencias pertinentes", en relación a una intención de lo que esté suponiendo el ser humano. Por otro lado, la autora indica las siguientes ventajas entorno al chatbot para Facebook:

- Permiten conectar con los usuarios en uno de los medios que más usan.

- Dentro de este tipo de herramientas, Messenger es la más popular.

- Dan la posibilidad de atender a miles de clientes a la vez.

- $\quad$ Son una de las maneras más eficaces de atraer la atención de la audiencia y lograr conversiones.

- Mejoran la personalización en la atención al cliente.

Según como lo describe la autora, las ventajas de un chatbot se pueden considerar, como elementos disruptivos dentro de la cognición de una sociedad y se pueden maximizar al estar relacionados con las redes sociales, dentro de las que destaca Facebook, lo que permitiría influir en una cantidad considerable de usuarios al estar activos y con ello romper paradigmas de comportamientos tradicionales dentro y fuera del Internet.

Por otro lado, en este proyecto se desarrolló un chatbot para la red social Facebook, sin olvidar que se podría implementar para otras redes sociales o plataformas como Skype, Telegram, Viper, Slack, Kik, Telephony, entre otras.

Para concluir, el conocimiento y el proceso cognitivo, sólo tienen que ver con el ser humano y se da en mayor medida en sociedad, por lo tanto, un robot, sería muy difícil que aprendiera por sí sólo cuestiones complejas y mayormente más complicado que aprendiera lo de la sociedad y más aún, la historia que ha acontecido a través del tiempo a la humanidad. 
Sin embargo, sí se le puede considerar como un ente con una aplicación embebida automatizada con inteligencia artificial, que podría subsanar y resolver satisfactoriamente con un alto grado de exigencia algunos procesos (consulta de información, compras de productos, automatización de sectores productivos, entre otros).

\section{Metodología}

Para desarrollar esta aplicación que será destinada en su mayoría a los millenials se utilizó las siguientes herramientas:

1. Dialogflow, esta herramienta indica en su sitio oficial que se desarrollan experiencias y que ofrecen a los usuarios nuevas formas de interactuar con su producto mediante la creación de interfaces conversacionales de voz y texto (un chatbot), con tecnología de inteligencia artificial; dentro de su diversidad de elementos que se encuentran en esta aplicación están los intentos, que son peticiones que los usuarios hacen cuando están interactuando con la aplicación (esperan un comportamiento predeterminado), otro elemento, es la integración, es aquel elemento que sirve para que se enlace con alguna plataforma de las mencionadas anteriormente. Existen dos versiones una gratuita y una empresarial.

2. Facebook para desarrolladores, es la herramienta que hace que los programadores de empresas, FreeLancer o cualquier persona inmersa en la computación se vuelva adicto a Facebook. A través de esta herramienta se hace un enlace entre ella y la herramienta Dialogflow (Figura 3). Es de suma importancia comentar que con esta herramienta se debe solicitar autorización de la aplicación que se está desarrollando al staff de Facebook, si esta no se autoriza los usuarios no podrán ver la aplicación y mucho menos interactuar con ella.

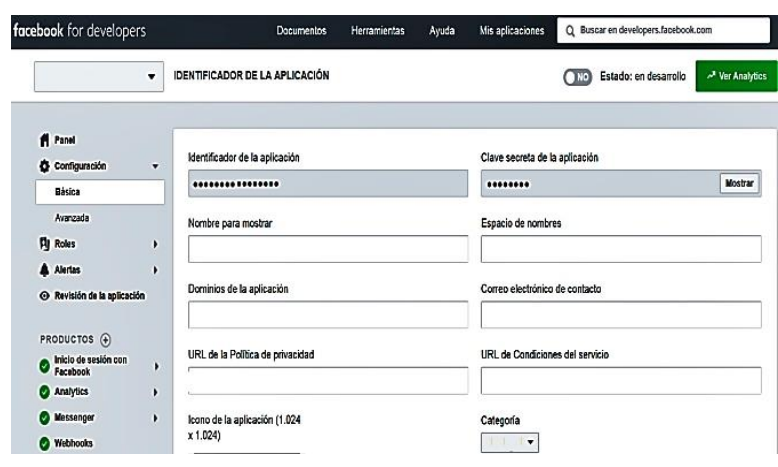

Figura 3 Configuración de Facebook con la página creada y la herramienta DialogFlow (Facebook,2018)

3. Botsociety, se describe en su página oficial como: una herramienta de diseño para Messenger que crea rápidamente una vista previa de alta fidelidad de un robot o un asistente de voz, con ella se hace una diagramación del proceso de interacción con el bot (Figura 4).

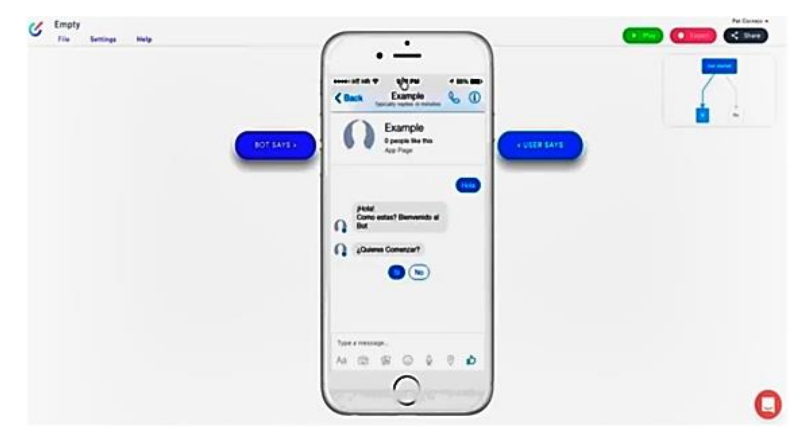

Figura 4 Maquetación del flujo conversacional Fuente: BootSociety, 2018

Dentro de la diversidad de herramientas que el gigante tecnológico llamado Facebook creó para desarrolladores las cuales son puestas para uso de los programadores, se encuentran dos de gran importancia dentro de la plataforma de Messenger:

1. Los componentes de Integración que según Facebook son: "un conjunto de funciones diseñadas para ofrecer todo lo que se necesita para crear fantásticas experiencias interactivas en Messenger, tales como API, plugins para web y una vista web completa". Todos estos elementos son realmente fantásticos hay algunos que sobresalen, Facebook los describe de la siguiente manera:

a) Webhooks. El webhook es un extremo HTTPS único (generalmente /webhook) que se expone y que acepta solicitudes POST. Es aquí donde el bot procesa y responde a todos los eventos de webhook entrantes. 
b)

NPL. La función de procesamiento de lenguaje natural es integrada en la plataforma de Messenger e incorpora la potente plataforma de lenguaje natural Wit.ai (impulsa la creación de aplicaciones y dispositivos con los que puede hablar o enviar mensajes de texto) directamente en los bots de Messenger, para que se pueda detectar la intención y el significado de los mensajes que se le envían. Cuando la función de NLP integrada se encuentra habilitada, la plataforma de Messenger devuelve el análisis de Wit de forma automática al webhook con cada mensaje.

2. Las conversaciones son estructuras complejas de mensajes de texto a las que se le puede incluir contenido multimedia, aunado al conjunto de opciones como plantillas, respuestas rápidas, botones, entre otras.

\section{Aplicación}

La figura 5, muestra la vista de cómo se presenta la herramienta a los usuarios de la red social Facebook cuando desean interactuar con la aplicación, en ella se presenta en la opción de enviar mensaje que está en color azul.

Es importante recalcar que la comunicación es en tiempo real (síncrona), de tal suerte que la herramienta ayudará al usuario con posibles respuestas para favorecerlo en la búsqueda de información.

El caso de estudio es la Universidad Politécnica del Valle de México (Upvm). Específicamente el Programa Educativo de Ingeniería en Informática.

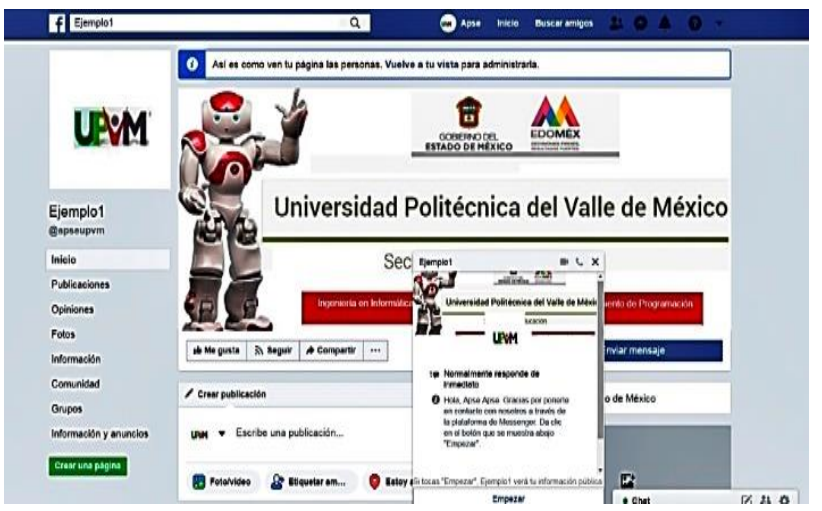

Figura 5 Antes de Iniciar el Chatbot
Las figuras 6 y 7 , muestran una interacción entre los usuarios de la red social y el bot, este chat ayuda al usuario en el trascurso de búsqueda de información, es importante recalcar que es un proceso de comunicación síncrona entre la aplicación y los usuarios, sin embargo, puede ser asíncrona cuando la plática es enviada automáticamente al buzón del administrador de la aplicación.

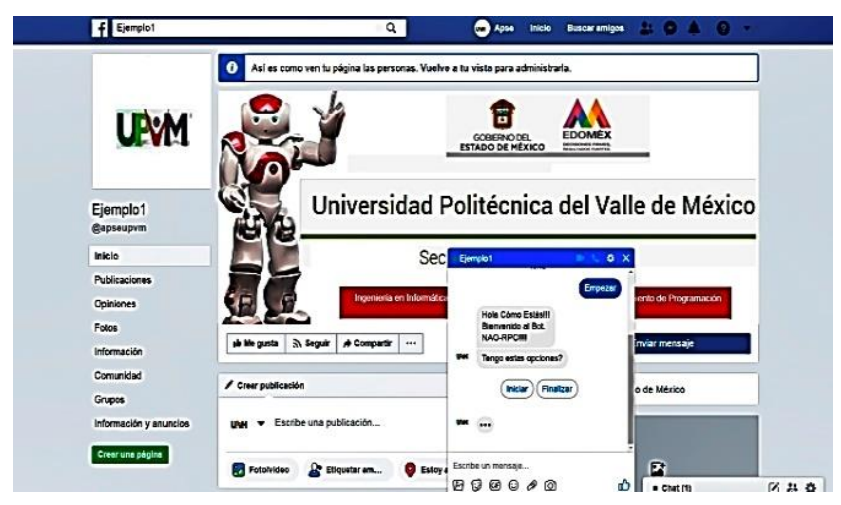

Figura 6 Inicio del Chatbot

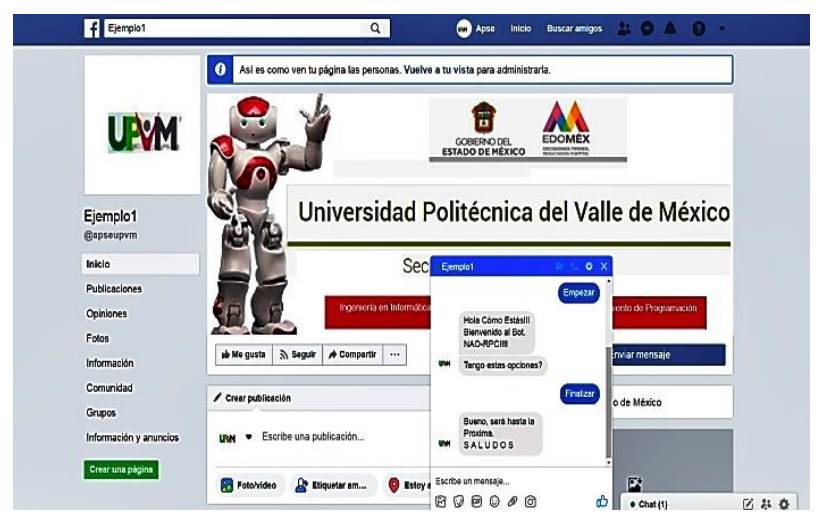

Figura 7 Salida del Chatbot

Cuando al usuario se le presenta el menú, sólo tendrá que dar clic en la opción que escoja y el bot lo mandará al receptor de mensaje e interpretará su decisión.

\section{Desarrollo de la Herramienta}

Para el desarrollo de la herramienta se tuvieron que crear varios comportamientos específicos (intentos) para que se pudiera interactuar con el bot, dentro de los cuales se encuentran los siguientes: 1. Carreras, 2. Becas, 3. Reinscripciones, 4. Posgrados, 5. Normatividad, entre otros. La figura 8, muestra el inicio del bot después de indicar la palabra iniciar, la cual presenta un menú con tres opciones.

La figura 9. Muestra la información respectiva a qué tipo de becas tiene la Upvm. 


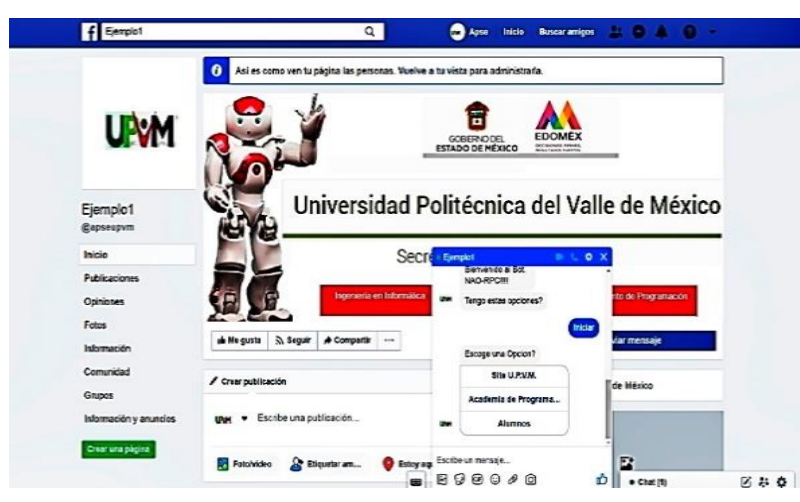

Figura 8 Primer menú del Chatbot

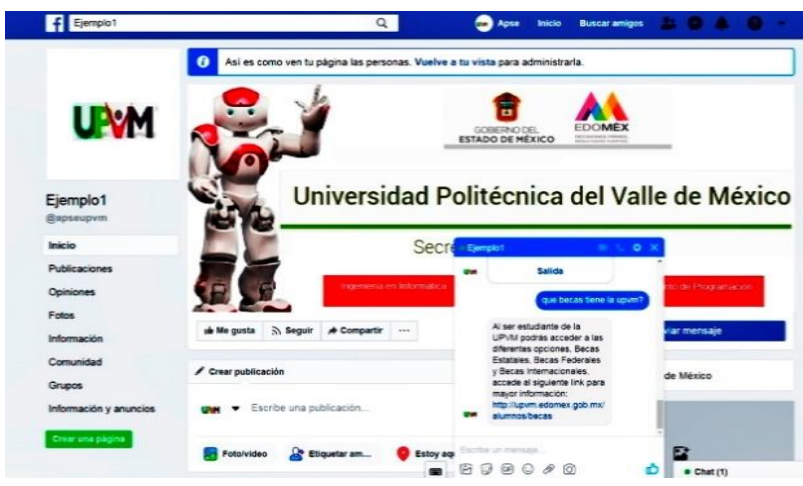

Figura 9 Solicitud de becas

Las figuras 10 y 11 , muestran la oferta educativa de la Upvm, que se compone de 8 Ingenierías y 1 licenciatura, las opciones se presentan a través de un menú desplegable con corrimiento a la derecha o a la izquierda según sea el caso, para que el usuario pueda escoger la carrera que busca y darle clic a ella.

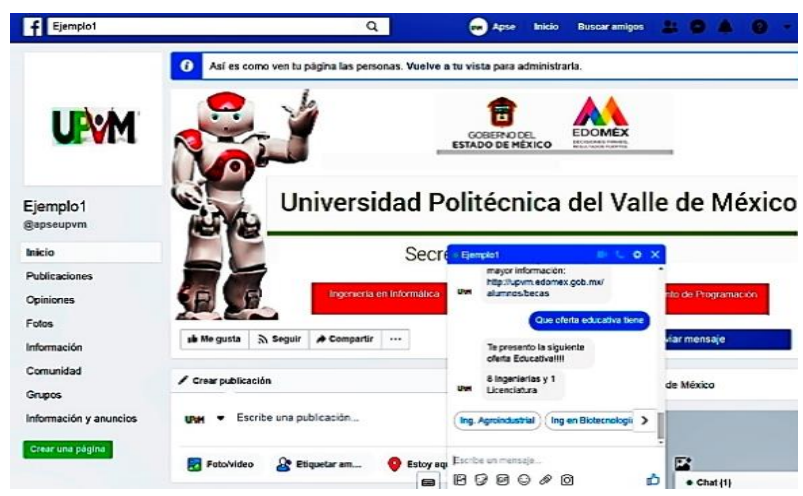

Figura 10 Respuesta a solicitud de Carreras

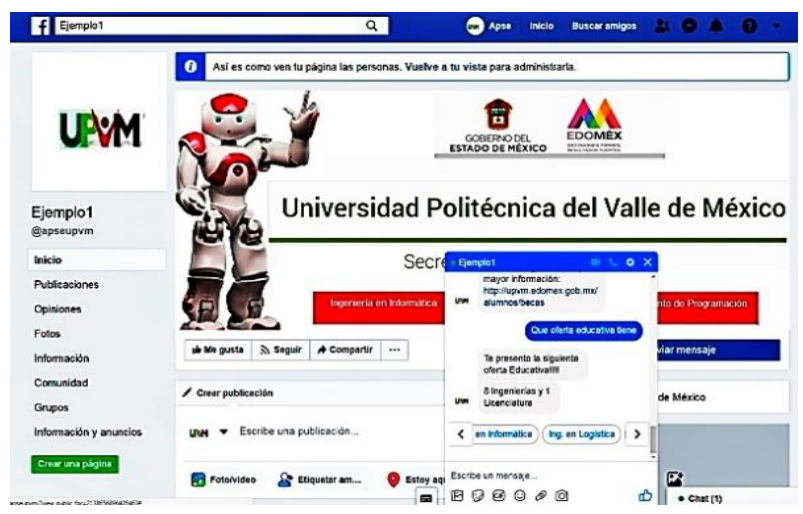

Figura 11 Respuesta a solicitud de Carreras (Cont.)

ISSN 2531-2197

ECORFAN ${ }^{\circledR}$ Todos los derechos reservados
Por último, procesos de importancia para los estudiantes son el de tutorías Figura. 12 y el de asesorías Figura. 13. Los cuales contienen información pertinente durante la estancia académica de ellos.

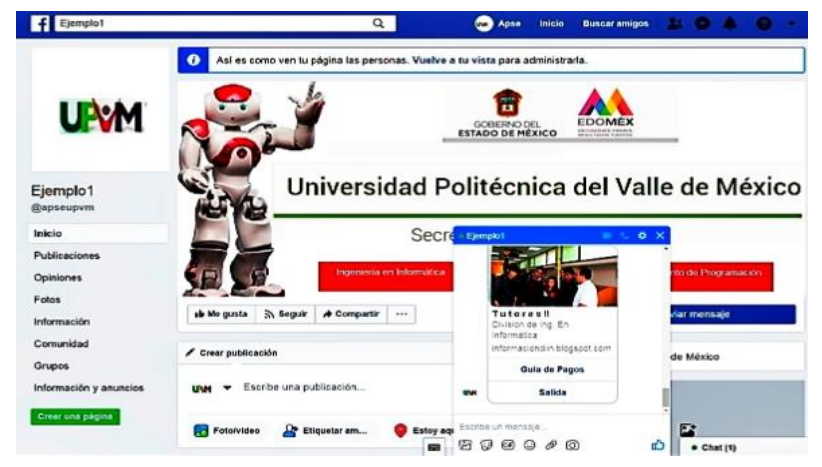

Figura 12 Respuesta a solicitud de tutores.

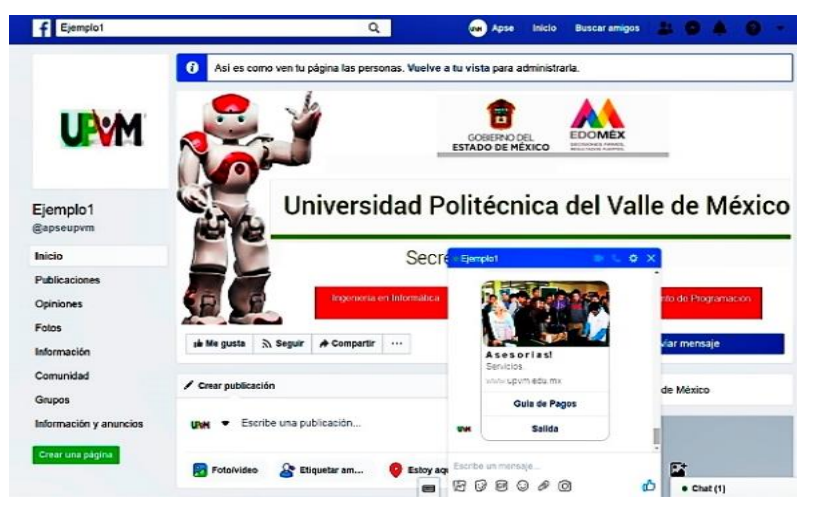

Figura 13 Respuesta a solicitud de Asesorías.

Las figuras anteriores son sólo una parte de la información que se presenta a los alumnos de licenciatura y posgrado e inclusive a los candidatos a ser alumnos. Por otro lado, también puede ser que el chatbot, no entienda alguna petición del usuario para ello existen los intentos fallidos (fallback), que se activan a una respuesta no exitosa a una petición. Ejemplos de dos respuestas a un intento fallido serían las siguientes: 1 . No entendí eso. ¿Puedes decirlo otra vez? y 2. Perdón, ¿Qué fue eso?

\section{Parte de Código del chatbot}

El código dentro del chatbot es una de las partes más esenciales ya que con él se pueden crear diversas plantillas para ser presentadas al usuario, a través del uso de botones, menús, imágenes, entre otros elementos. Adicional se puede agregar diversas respuestas a una petición, el código que se esquematiza en la figura 14, describe de manera general lo que debe tener una plantilla: Attachment, Type y Payload, son instrucciones para que puedan ser reconocidas por alguno de los integradores (Plataformas) que ya se habían mencionado, Facebook, Skype, Twitter, entre otros.

PÉREZ-CALDERÓN, Ricardo \& SUAREZ-JACOBO, Martin. La cognición en la web a través de un chatbot como elemento disruptivo. Revista de Tecnología Informática. 2019 


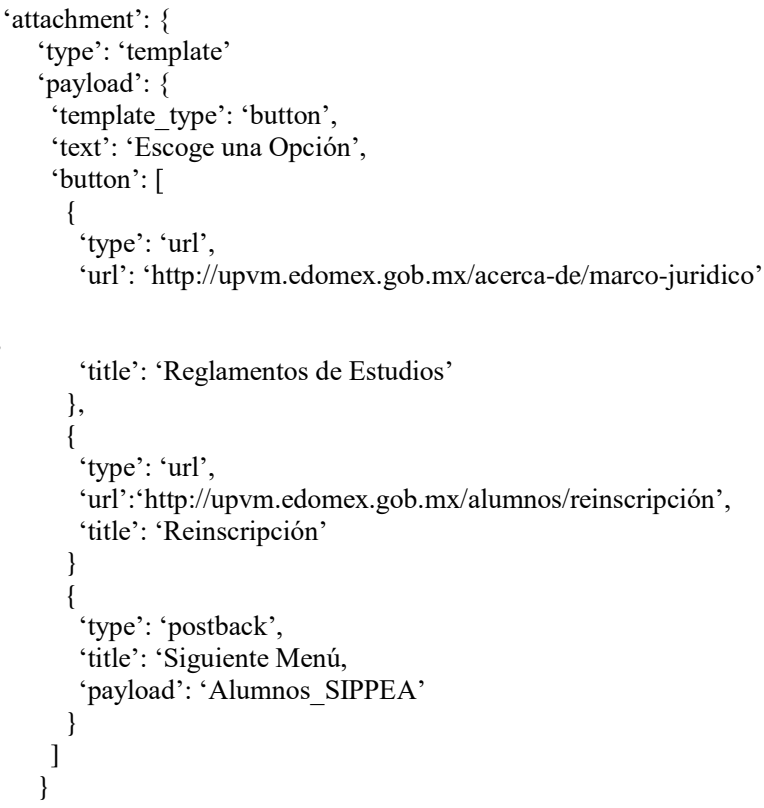

Figura 14 Código genérico de un intento

\section{Resultados}

La herramienta se puso a prueba con una muestra piloto de diversos alumnos tanto del programa de Ingeniería en Informática como ajenos al programa de estudios (Ingeniería Industrial, Licenciatura en Administración y gestión de Empresarial, entre otras). Después de usar la herramienta se les aplicó un cuestionario y los resultados fueron los siguientes:

Ítem 1. ¿Le ayudó el uso del chatbot en la búsqueda de información?

El $88 \%$ de los entrevistados indicaron que sí les había ayudado en la búsqueda de información, el $12 \%$ indicó que todavía falta más robustez en la herramienta. (Gráfica 1).

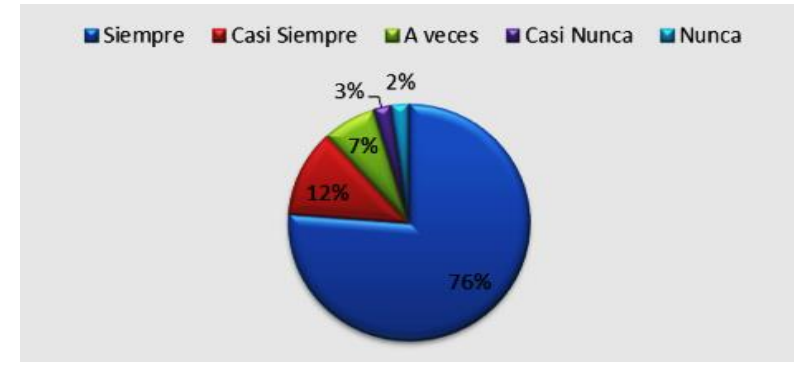

Gráfico 1 ¿Le ayudó el uso del chatbot en la búsqueda de información?

Ítem 2. ¿El chatbot serviría para mejorar el impacto en un uso comercial, es decir en la imagen de la empresa?

El $98 \%$ de los encuestados indicaron que sí puede servir para mejorar el impacto en el uso comercial, el $2 \%$ indicó que no es así. (Gráfica 2).

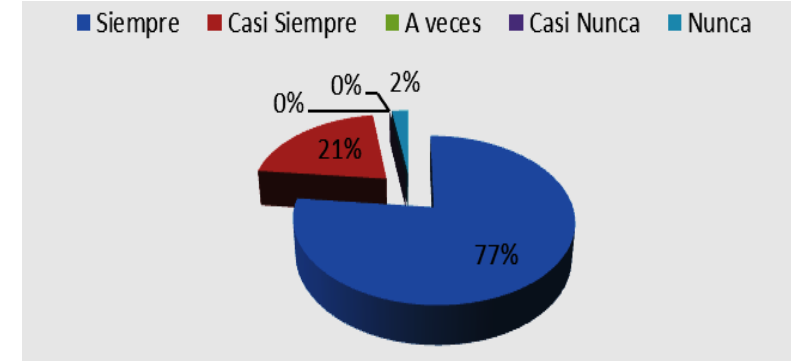

Gráfico 2 ¿El chatbot serviría para mejorar el impacto en un uso comercial, es decir en la imagen de la empresa?

\section{Ítem 3. ¿El uso de los chatbot generaría credibilidad, en los usuarios?}

El $88 \%$ de los usuarios indicaron que si puede mejor la credibilidad de las empresas en la atención a los clientes, el $12 \%$ todavía está indeciso. (Gráfica 3).

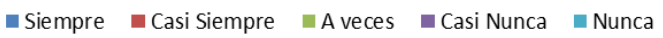

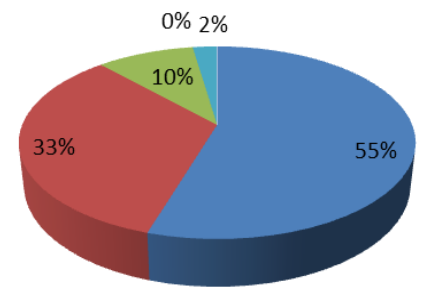

Gráfico 3 ¿El uso de los chatbot generaría credibilidad, en los usuarios?

Ítem 4. Un elemento disruptor es algo que cambia el paradigma de comportamiento, ¿Esta herramienta sería un elemento disruptivo?

El $88 \%$ de los usuarios consideran que el uso de un chatbot sí es un elemento tecnológico para cambiar el paradigma de comportamiento en cuanto al manejo de plataformas en Internet, el $12 \%$ no lo considera así. (Gráfica 4).

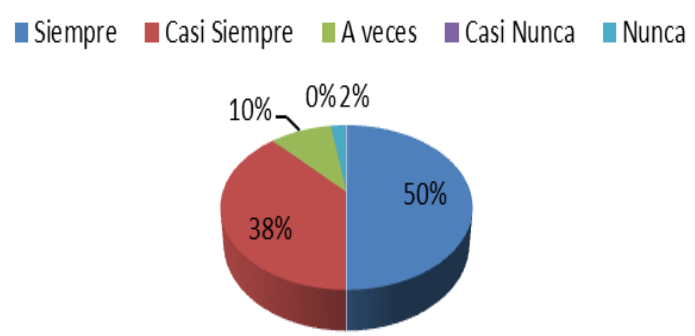

Gráfico 1 ¿Esta herramienta sería un elemento disruptivo?

Ítem 5. ¿Qué tanto recomendaría el uso de este un chatbot, con sus contactos?

El $91.5 \%$ de los usuarios sí recomendarían el uso de este chat para cuestiones académicas, el $8.5 \%$ de los usuarios no lo recomendaría. 


\section{Ítem 6. ¿Le fue complicado el uso de esta herramienta?}

El $71 \%$ de los usuarios indicaron que no fue complicado el uso de esta herramienta, el $29 \%$ de los usuarios si lo fue, debido a que no sabían qué preguntar o cómo preguntar.

\section{Ítem 7. ¿Qué red social usa en Internet?}

El $81 \%$ de los usuarios usan la red social Facebook, un $6 \%$ usa Instagram y el otro $6 \%$ no usa redes sociales. Por lo tanto, este proyecto sí tiene una focalización acertada en el uso de la red social de mayor demanda como lo es Facebook.

\section{Ítem 8. ¿Ha usado otros chatbot?}

El $44 \%$ de los usuarios sí ha usado o interactuado con otros chats, el $56 \%$ de los usuarios no ha usado ningún chatbot.

La tendencia es a crecer en los próximos años como lo indica la organización Pew Research Center.

\section{Ítem 9. ¿Cuáles?}

Los usuarios comentaron que sí han usado este tipo de herramienta y mencionan a los siguientes chatbots: Santander, GEM, Word intranet, Xbox en Facebook, becas, Asistencia foros, weexx, ayuda en Bancos, Cedco, Coppel, Cinepolis y por último, como compra de boletos en aerolíneas. Se observa, que el uso de los chatbots no es tan desconocido por los usuarios y la tendencia de las empresas es hacer uso de esta tecnología.

\section{Ítem 10. ¿Cuál es su ocupación?}

El $81 \%$ de los usuarios son estudiantes, el $5 \%$ de ellos son docentes y el $14 \%$ de ellos son empleados. Efectivamente serán los millenials lo que acabaran con abarcar las ofertas laborales de las empresas.

\section{Conclusiones}

El proyecto se encuentra en fase de aprobación por parte del staff de Facebook debido a que se tiene que cumplir con las políticas de desarrollo de dicha empresa, aunado a que también está en fase de autorización por parte de la Rectoría de la Universidad.
Sin embargo, la prueba que se realizó al conjunto de usuarios ajenos al Programa Educativo, evidenció que es una herramienta innovadora y que en conjunto con el staff de desarrollo de la página Web de la Institución se obtienen resultados muy alentadores en los diferentes ámbitos que la institución persigue, por ejemplo, ayuda a los estudiantes en su necesidad de búsqueda de información en las diversas áreas de la Universidad, es decir, en el proceso de reinscripción de cada cuatrimestre (fechas, costos, requisitos) o en otros procesos como: normatividad, tutorías, asesorías, entre otros e incluso ayudará a la Institución a la captación de matrícula de los diferentes Programas Educativos de Licenciatura y Posgrado o en Educación continua (cursos de idiomas o cursos tecnológicos).

Por último, la Upvm ya cuenta con una aplicación muy limitada (si se le hace un cuestionamiento, hasta un día de posterior se obtiene la respuesta, y si de nuevo se hace otra petición será hasta el siguiente día para obtener la respuesta). Por todo lo anterior, se puede concluir que las instituciones públicas o empresas privadas están observando la tendencia del uso de este tipo de tecnología. Sin duda alguna un chatbot mejoraría la atención y satisfacción de los clientes de cualquier organización. El análisis del uso será hasta que se libere la versión en producción.

\section{Recomendaciones}

Es necesario pagar la versión extendida de DialogFlow para poder interactuar con otras características de la plataforma como sería la voz e incluso para garantizar la migración de las APIs de la misma empresa.

Hacer la prueba de la aplicación en al menos dos redes sociales más, para garantizar el funcionamiento del chatbot.

\section{Referencias}

Araya, Natalia (2014), En términos concretos la cognición son el desarrollo de habilidades del pensamiento que el ser humano trae desde su nacimiento y en su mayoría se aplican a circunstancia propias de la vida, recuperado de http://www.scielo.sa.cr/pdf/aie/v14n2/a03v14n2 .pdf, para su consulta el día 02 de abril del 2018. 
Campos H. Miguel, (2004), Una aproximación sociocultural a los procesos cognoscitivos en el contexto educativo, Perfiles Educativos, vol. XXVI, núm. 104, tercera época, pp. 7-32, Instituto de Investigaciones sobre la Universidad y la Educación Distrito Federal, México.

Gómez, Zaira (2015), Procesos cognitivos en UX, http://revista.uxnights.com/sobre-procesoscognitivos-en-ux/, recuperado el día 02 de mayo del 2018.

Gutiérrez-Rubí, Antoni, (2014), recuperado de: https://www.forbes.com.mx/6-rasgos-clave-delos-millennials-los-nuevos-consumidores/, el día 20 de Enero de 2018, para consulta.

https://www.eleconomista.es/tecnologia/noticia s/7475048/04/16/Los-chatbots-de- inteligencia artificial-el-futuro-de-las-apps-de-

mensajeria.html, recuperado el día 12 de Diciembre del 2018 para su consulta.

https://www.eleconomista.es/tecnologia/noticia s/7468203/04/16/El-Washington-Post-

empleara-chatbots-para-la-difusion-de-sus-

contenidos.html, recuperado el día 12 de Diciembre del 2018 para su consulta.

https://www.iadvize.com/blog/es/libro-blancocomo-transforman-los-chatbots-el-customerexperience-online/, recuperado el día 20 de Julio del 2017 para su consulta.

Charlan, J, (2018), Qué es un chatbot y para qué sirve, recuperado, de: https://www. esic.edu/rethink/2018/08/04/que-es-un-chatboty-para-que-sirve/, el día 15 de Septiembre del 2018 para consulta.

Hervouet Julien, (2019), The Total Economic Impact Of ibbü By iAdvize, recuperado de https://info.iadvize.com/hubfs/TEI\%20of\%20i Advize\%20ibbu \%CC\%88\%20\%20online\%20retailer.pdf, el día 1 de Marzo del 2019 para consulta.

Nathan, Shedron, (2014), Information Interaction Design: A Unified Field Theory of Design, recuperado de: http://nathan.com/information-interactiondesign-a-unified-field-theory-of-design/, el día 15 de abril del 2018 para consulta.
Pew Research Center, (2010), Millennials, recuperado de: https://www.pewresearch.org/topics/millennials /, el día 14 de Junio del 2018 para consulta

Revista Digital Infocop de España, (2011), El Efecto Google y su Influencia en los Procesos Cognitivos, recuperado de: http://www.infocop.es/view_article.asp?id=360 1, el día 03 de abril del 2018 para consulta.

V Aliaga Gálvez, G. H., Abarca, A., \& Arturo, J. (2019). Aplicación móvil para diagnosticar posibles fallas automotrices utilizando la herramienta IBM WATSON para la empresa VECARS \& TRUCKS SAC.

Vergara Gabriel, (2008), Curso de Psicología social, (2008), Construcción social de la realidad, recuperado de: http://psicosocialrecuperado de: uv.blogspot.mx/2008/07/62construccin-social-de-la-realidad.html, el día 1 de Abril del 2018 para consulta.

Ventura Berta, (2018), ¿Qué son los chatbots de Facebook?, recuperado de: https://www.cyberclick.es/numerical-blog/queson-los-chatbots-de-faceboo-la-nueva-granapuesta-de-zuckerberg,el día 1 de julio del 2018 para consulta. 